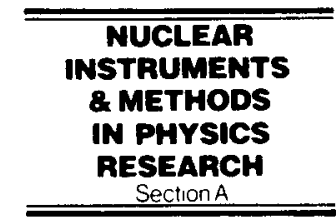

ELSEVIER

\title{
Evaluation and measurement of the experimental gamma-ray peak broadenings and recovery times in a gain stabilized system
}

\author{
D. O'Connor ${ }^{\mathrm{a}, *}$, G. Knoll ${ }^{\mathrm{a}}$, R. Ziff ${ }^{\mathrm{b}}$ \\ ${ }^{a}$ Department of Nuclear Engineering, Universtty of Michigan, Ann Arbor, Michigan 48109, USA \\ ${ }^{b}$ Department of Chemical Engineering, University of Michigan, Ann Arbor, Michlgan 48109, USA
}

\begin{abstract}
Peak broadenings induced by gain wander in a gain stabilized system were measured and found to approximate values determined from prediction techniques and a system simulation. An equation is derived for the recovery time of the system; its predictions are validated by experimental measurements.
\end{abstract}

\section{Introduction}

For optimum results in gamma-ray spectroscopy it is imperative that all detector pulses experience a constant level of amplification (gain) before arriving at the multichannel analyzer (MCA). Factors such as a variation in temperature or voltage instabilities can introduce gain drifts into the pulse processing system. Devices known as gain stabilizers employ feedback techniques to maintain a constant system gain. The system is defined as the detector and all of the electronic components. As a result of the inherent statistical nature of the detection process, gain stabilizers can introduce gain adjustments when no adjustments are necessary. The overall effect of the unwarranted gain adjustments, known as gain wander or peak hunting, may broaden the experimental gamma-ray spectrum peaks and consequently lead to a loss of pulse height resolution. In the event of an actual gain shift, the peak will be unavoidably broadened during the period of time it takes the gain stabilizer to correct the system's gain. The recovery time, discussed later in the paper, is one measure of the length of time required for gain correction.

Variations in the system gain can be determined by tracking the centroid channel position of a well defined reference peak in an experimental gamma ray spectrum. A common type of gain stabilizer operates by monitoring three sets of MCA channels (windows) which span a reference peak, as shown in Fig. 1. The gain stabilizer will alter the gain of the system by one gain increment if a reference pulse is recorded in either the upper or lower window. For example, the appearance of a pulse in the upper window indicates a possible increase in the system gain, therefore, the gain is reduced by one gain increment.

\footnotetext{
* Corresponding author.
}

To the gain stabilizer, center window pulses are essentially inert and indicate that the system gain has remained unchanged. The system gain is limited to a set of discrete values, known as gain states, consisting of integer multiples of the gain increment added to the original set gain state. The resolution of a gain stabilizer is defined as the width of a MCA channel measured in units of gain increment.

An approximately Gaussian distribution of pulse amplitudes is produced by the instruments employed to detect gamma rays. Thus, without a gain drift, there remains a probability that a reference pulse will be binned into one of the upper or lower window channels. Such a window pulse will cause the gain stabilizer to undertake corrective action in response to an apparent but nonexistent gain drift. In the absence of any actual change in the system gain, the width of the stabilized reference peak can be described as, $\sigma_{\text {stablized reference peak }}^{2}=\sigma_{\text {unstabllized reference peak }}^{2}+\sigma_{\text {gain wander }}^{2}$.

\section{Prediction techniques}

We have previously presented two new techniques, the eigen value solution and the analytic moment solution, for the prediction of the experimental gamma-ray peak broadening induced by gain wander in a gain stabilized system [1]. They are summarized below.

Whenever the current system gain state has a value greater than the set state, then the probability of a decrease in system gain is greater than that for an increase. The eigen equation incorporates a matrix which contains the transition probabilities, for each possible gain state, that the next reference pulse will cause the system gain state to be increased, decreased or remain unchanged. The matrix 


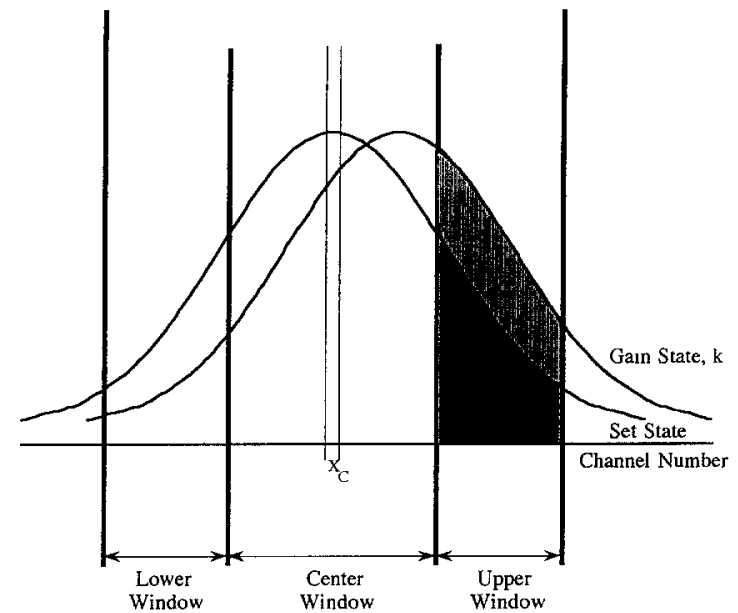

Fig. 1. The gain stabilizer defines three windows that span the reference peak. The gain stabilizer will alter the gain by one gain increment for every pulse which is placed in either the upper or lower window. An upper window pulse indicates an increase in gain, thus, the system gain is reduced by one gain increment. The probability $P_{\mathrm{U}}$ that the next reference pulse will cause the gain state to be decreased is denoted by the area under the Gaussian curve delimited by the upper window.

is tri-diagonal as the alteration in gain state is always only one increment, regardless of the amplitude of the reference pulse initiating the change. The eigen solution [1] solves the eigen equation for an eigenvector, known as the state vector, which describes the distribution of the probabilities that the system is in a specific gain state. Because the transition matrix is derived from a Gaussian distribution, the state vector is also Gaussian. The magnitude of the reference peak broadening resulting from the inherent gain wander can be determined from the FWHM of the converged distribution of the state vector.

The analytic moment solution [1] utilizes the moment equations and the error-function to derive an equation to predict the experimental gamma-ray peak broadening induced by gain wander. For each gain state, the area bounded by the reference peak and the channels delimiting a gain stabilizer window defines the probability that the amplitude of the next pulse will cause the MCA to place it into a specified window. The first two terms of the error function are used to describe the Gaussian distribution of reference pulse heights so that a general gain state probability equation is developed. Recall that by definition, the second moment of the distribution of gain states (pulse amplitudes) is the variance. Therefore, it is possible to evaluate the broadening of the reference peak due to gain wander without any iterations by using Eq. (11) in Ref. [1]. The only variables necessary for the calculation of the variance of the gain stabilized reference peak are the FWHM of the unstabilized peak, the gain stabilizer resolution, and the number of MCA channels defining the center window.

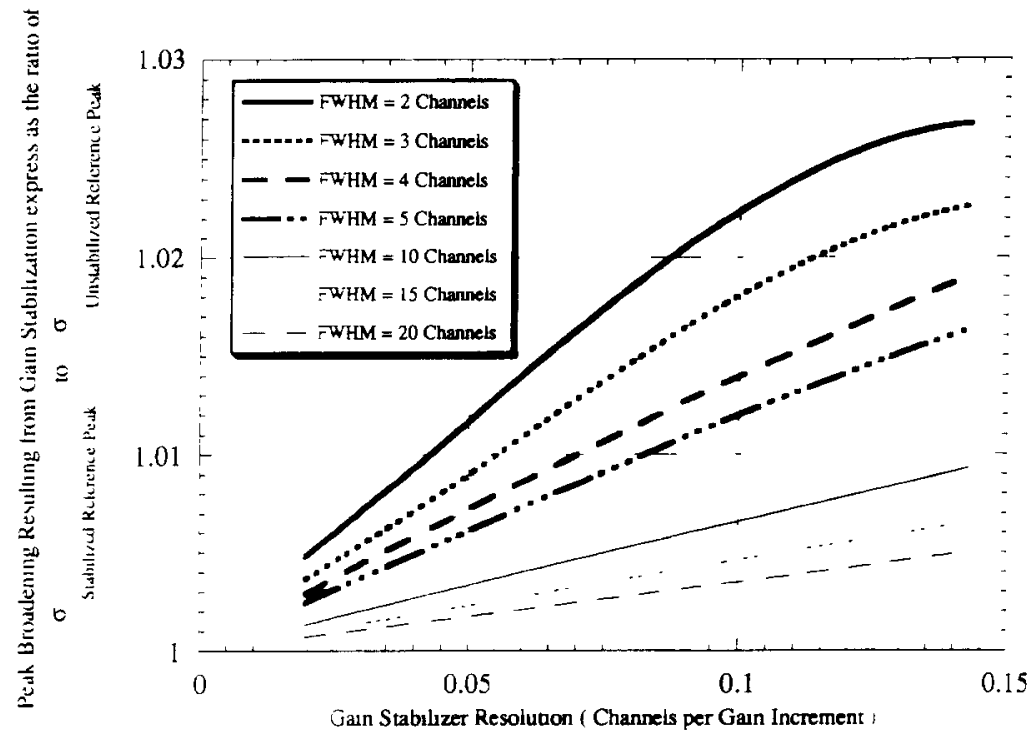

Fig. 2. Broadening of the reference peak that results from gain wander in a gain stabilized system as determined by the eigenvalue solution. The center window width is defined as one channel, the centroid channel of the reference peak. The upper and lower windows extend from the center window to a width of two standard deviations. 


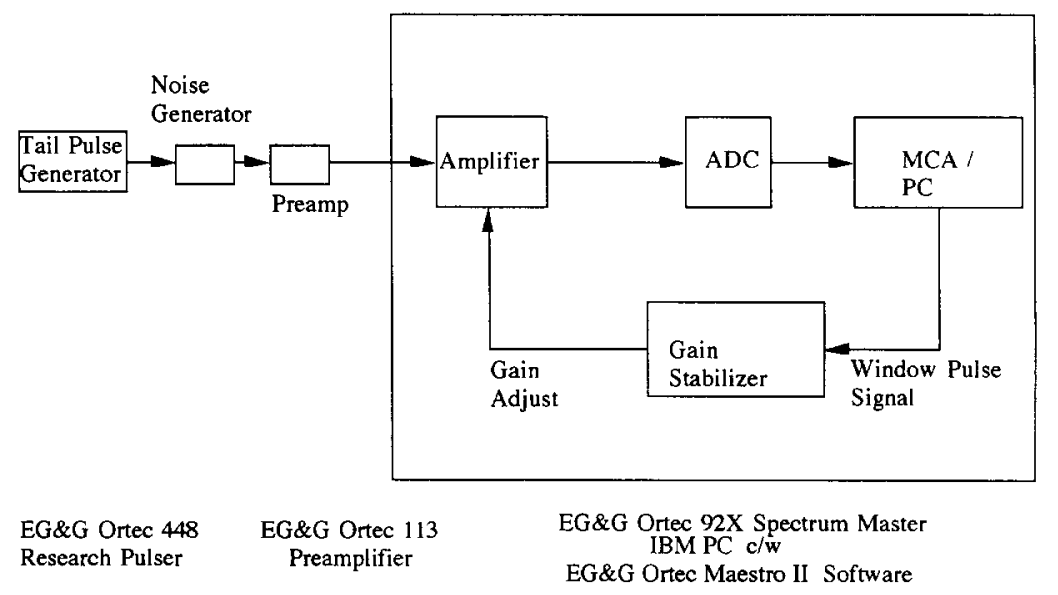

Fig. 3. Pulse processing system for experimental determination of the broadening of the stabilized reference peak induced by gain wander.

For comparative purposes, the recursive solution proposed by Ferguson [2] was revised to include a Gaussian distribution of reference pulse amplitudes. The revision has also been previously described [1]. Predictions of all three models are consistent considering the approximations made in their derivations. As an example, Fig. 2 displays the ratio of the width of the stabilized peak to the unstabilized peak as determined by the eigen solution. For the calculations the center window was described as one channel which is the most common choice in commercial stabilizers. In this example the upper and lower windows extend from the center channel out to two standard deviations of the width of the unstabilized reference peak.

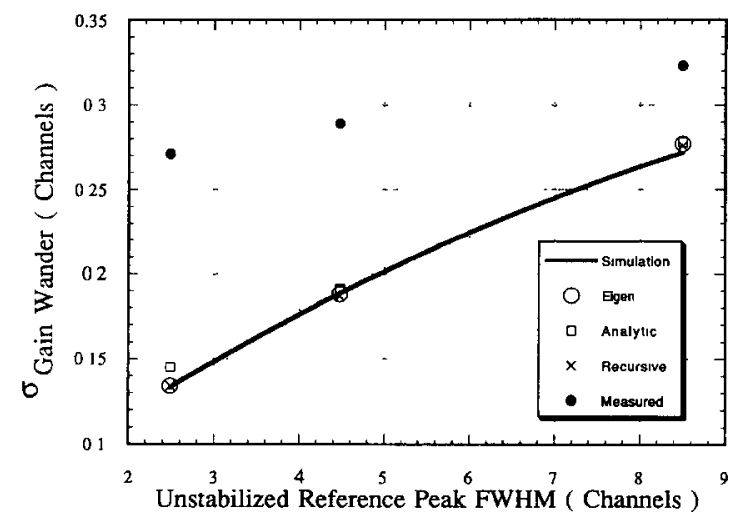

Fig. 4. Comparison of peak broadenings induced by gain wander in a gain stabilized system as determined by the prediction techniques, the simulation and experimental measurements. The gain stabilızer resolution was set at 0.03738 channels per gain increment.

\section{Experimental measurements of peak broadenings}

The pulse processing system displayed in Fig. 3 was used to measure the experimental peak broadening induced by gain wander. A Gaussian distribution of reference pulse amplitudes was created by passing the output of a tail pulse generator through a specially designed noise generator. A variable noise level in the noise generator allowed the FWHM of the reference pulse distribution to be varied. To eliminate the major causes of gain drift, the temperature of the environment surrounding the system was held nearly constant and the line voltage supplying the system was filtered through a stabilizer.

The peak broadening due to gain wander was determined as follows: first, the FWHM of the reference pulse distribution was recorded when the gain stabilizer was not operating. The measurement was taken over a short period of time so as to minimize any chance of gain drifts. The FWHM of the reference pulse distribution was then remeasured with the gain stabilizer functioning. The peak broadening induced by gain wander, displayed in Fig. 4, was then unfolded using Eq. (1). The experimental values are approximately $40 \%$ larger than analytic results. This is only slightly better agreement than the figure of $50 \%$ which Poulsen [3] reports in a 1991 comparison of previously published prediction techniques $[2,4,5]$ to data collected by gain stabilized pulse processing systems designed for atmospheric and space studies.

\section{System simulation}

Due to equipment limitations, the range of experimental data was restricted to a single gain stabilizer resolution 
value and reference peak widths of under 10.0 channels. A Monte Carlo simulation of the gain stabilized pulse processing system was developed to test the applicability of the prediction techniques over a greater range of possible settings.

The stimulation samples and stores individual reference pulses from a Gaussian distribution of set FWHM. The reference source can be defined as either a pulser and noise generator combination similar to the experimental setup described above (constant time intervals between pulses) or a radioactive source (random time intervals between pulses). The reference pulse is then amplified by the current system gain state before being presented to the MCA for binning. Any number and width of gain stabilizer windows can be set. If the reference pulse is binned into an upper or lower window then the stabilizer will alter the system gain by one increment of appropriate sign. The implementation time of the gain increment is defined by the user. At the end of a specified period of time or number of events, the value of the stabilized reference peak width FWHM is calculated from the binned MCA data by calculating moments. The unstabilized reference peak width is determined by multiplying the collected unamplified reference pulse distribution and the constant gain of the set state. Moments are again used to determine the FWHM. The peak broadening due to gain wander is calculated by the quadrature subtraction of the unstabilized reference pulse distribution width from the stabilized value as suggested by Eq. (1). The excellent agreement between the simulation results and the values determined by the prediction techniques is shown in Fig. 4.

\section{Evaluation of recovery time}

A sudden and real change in system gain will displace the system gain to some value other than the set state and thus lead to a potential loss of pulse height resolution. To determine the effectiveness of the gain stabilizer it is necessary to evaluate the period of time required for the system to compensate for the gain displacement and return the gain state to the set state (or some reasonable approximation of the set state). We have defined the term system recovery time as the length of time required for the system to recover from an initial displacement, $\alpha(t)$, of unity to a value of $1 / \mathrm{e}$. The displacement is defined as

$\alpha(t)=\frac{\epsilon(t)}{\chi}=\frac{\chi+z(\epsilon)}{\chi}$,

where $\epsilon$ is the centroid error, defined as the distance of the reference peak's centroid location from the set state's centroid location which corresponds to the system's drifted gain state at time, $t ; \chi$ is the distance of the reference peak's initial centroid displacement from the set state's centroid location which corresponds to the system's drifted gain state at time, $t=0 ; z(\epsilon)$ is the corrected distance at time $t$. All of the above distances are measured as a fractional value of the unstabilized reference peak's $\sigma$. An error value of $\alpha(t)=0$ describes the system without any gain drifts. For a centroid error $\epsilon(t)$ following an initial displacement, the average size of a correction is defined as,

$\overline{z(\epsilon)}=+\Delta p_{\mathrm{L}}(\epsilon)+(0) p_{\mathrm{C}}(\epsilon)-\Delta p_{\mathrm{U}}(\epsilon)$,

where $\Delta$ is the fractional channel width corresponding to one gain increment; $p_{\mathrm{L}}, p_{\mathrm{C}}, p_{\mathrm{U}}$ are the probabilities that the next reference pulse will be binned in the lower, center and upper windows, respectively. Recall that a lower window pulse indicates that the gain is drifting downwards and thus initiates a positive gain increment. The rate of change of the error rate is thus defined by,

$\chi \frac{\mathrm{d} \alpha}{\mathrm{d} t}=\Delta R\left[p_{\mathrm{L}}(\epsilon)-p_{\mathrm{U}}(\epsilon)\right]$,

where $R$ is the activity of the reference source. The unstabilized reference peak is assumed to be Gaussian distributed and is approximated by using the first two terms of the error function. Furthermore, it is assumed that the upper and lower gain stabilizer windows (see Fig. 1) extend from $a \sigma$ to infinity. Eq. (4) can then be rewritten as

$\frac{\mathrm{d} \alpha}{\mathrm{d} t}=\tau\left[\alpha^{3}-\zeta^{2} \alpha\right]$

where

$\tau=\Delta R k \chi^{2} / 6$,

$-\zeta^{2}=3\left(a^{2}-2\right) / \chi^{2}$

and $k=\pi^{-05}$, a constant derived from the error function. Rearranging Eq. (5) for integration yields

$\int_{1}^{\alpha} \frac{\mathrm{d} \alpha}{\alpha[\alpha+\zeta][\alpha-\zeta]}=\tau \int_{0}^{t} \mathrm{~d} t$

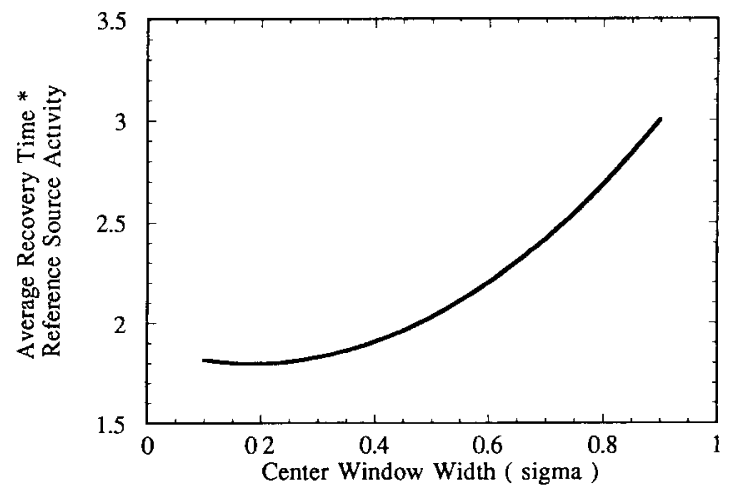

Fig. 5. Product of the average recovery time and the reference source activity plotted as a function of window width. The gain stabilizer resolution is set to 0.03738 channels per gain increment. 


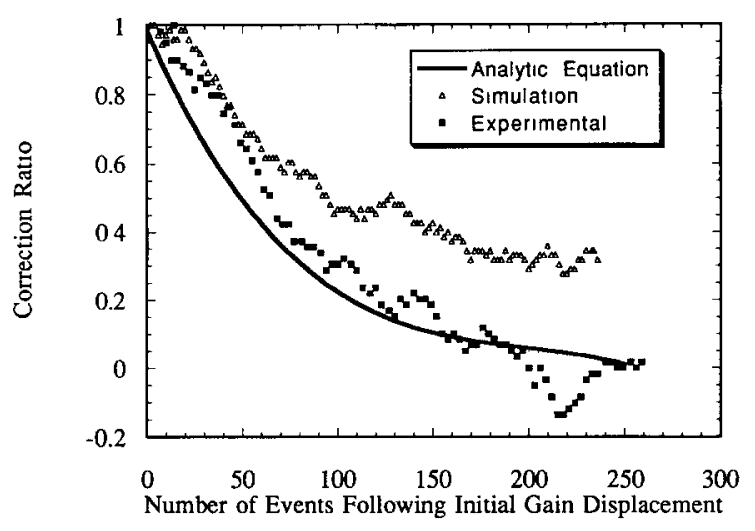

Fig. 6. Instantaneous location of the reference peak centroid following a displacement off the set channel of 2 channels. FWHM of the unstabilized reference peak is 6.58 channels, reference source activity $=5 / \mathrm{s}$. The gain stabilizer resolution is set to 0.02730 channels per gain increment.

Integrating Eq. (8) and rearranging yields the error rate as a function of time:

$\alpha(t)=\frac{\zeta}{\sqrt{1-\left[1-\zeta^{2}\right] \mathrm{e}^{2 \zeta^{2} \tau t}}}$.

The equation for recovery time, $t_{\mathrm{d}}$, is determined by rearranging Eq. (9) with $\alpha(t)$ set equal to $1 / \mathrm{e}$ :

$t_{\mathrm{d}}=\ln \left(\frac{1-\mathrm{e}^{2} \zeta^{2}}{1-\zeta^{2}}\right) /\left(\Delta R k\left(2-a^{2}\right)\right)$.

The recovery time, described by Eq. (10), shows only a slight dependence upon the initial displacement, $\chi$. Fig. 5 displays the unitless variable defined as the product of recovery time and the reference source activity, $R$, as a function of center window widths. The recovery times used in this plot were averaged over the initial displacement range of $0.1 a$ to $0.9 a$.

\section{Experimental measurements of recovery time}

Experimental measurements of the recovery times were undertaken with the previously described electronic pulse processing equipment. At a particular system gain, the corresponding value for the centroid of the reference peak was determined by collecting several unstabilized pulse height spectra. The set point of the gain stabilizer was then set equal to the unstabilized centroid value. The gain stabilizer was disengaged and the system gain was increased such that the centroid of the reference peak was altered from the set value. Once again, several unstabilized pulse height spectra were taken such that the initial displacement could be evaluated. The gain stabilizer was then engaged and the system gain was recorded as a function of time as the system compensated for the perceived gain shift, and the recovery time was measured. Monte Carlo simulations of the recovery from displacement were also carried out. As shown in Fig. 6, the predictions of recovery time are in close agreement with the simulation and the experimental values. The recovery time equation, as expected, predicts a smooth recovery that does not display the fluctuations seen from random sampling that is inherent in both the experimental measurements and the simulations.

\section{Conclusions}

A Monte Carlo simulation of a gain stabilized system was developed. The values for the gamma-ray peak broadening induced by gain wander in a gain stabilized system determined by the simulation were in very close agreement with the three prediction techniques discussed. For reasons we have not yet identified, the experimentally determined values of the peak broadening were larger than the predicted values, but the discrepancy is somewhat smaller than reported in a previous study [3].

The gain stabilizer recovery time was introduced as the length of time, following a gain shift, that is required for the displacement to decrease from 1 to 1 /e. An equation evaluating recovery time as a function of center window width and initial displacement was derived and predictions were made of the displacement as a function of time following a gain shift. Experimental values of the instanteous reference peak centroid location during the recovery period closely match the data predicted from the recovery time equation as well as the system simulation.

\section{Acknowledgement}

The authors express their gratitude to Dr. Tim Twomey of EG\& G Ortec for the loan of the equipment that was required for the experimental portions of this study.

\section{References}

[1] D. O'Connor, G. Knoll and R. Ziff, 1992 IEEE Nuclear Science Symp. Conf. Record, Orlando, FL, Vol. 1 (1992) p. 31.

[2] J. Ferguson, Nucl. Instr. and Meth. 58 (1968) 318.

[3] J.M. Poulsen, Instituto TESRE-CNR, Internal Report no. 155 (1991)

[4] R.A. Dudley and R. Scarpatetti, Nucl. Instr. and Meth. 25 (1964) 297.

[5] R.A. Dudley, Nucl. Instr. and Meth. 46 (1967) 116 